\title{
Smoking status and pathological response to neoadjuvant chemotherapy among patients with bladder cancer: a pooled analysis
}

\author{
Tongchen $\mathrm{He}^{\#}$, Jiao Hü, Dongxu Qiu, Hao Deng, Jian Hu, Jinbo Chen, Xiongbing Zu \\ Department of Urology, Xiangya Hospital, Central South University, Changsha, China \\ Contributions: (I) Conception and design: T He, J Hu; (II) Administrative support: D Qiu, H Deng; (III) Provision of study materials or patients: J \\ $\mathrm{Hu}$; (IV) Collection and assembly of data: J Chen, X Zu; (V) Data analysis and interpretation: T He; (VI) Manuscript writing: All authors; (VII) Final \\ approval of manuscript: All authors. \\ \#These authors contributed equally to this work. \\ Correspondence to: Jinbo Chen, Xiongbing Zu. Department of Urology, Xiangya Hospital, Central South University, Changsha, China. \\ Email: chenjinbo1989@yahoo.com; zuxbxyyy@126.com.
}

\begin{abstract}
Background: Smoking status has been confirmed as an independent prognostic factor for bladder cancer. However, for patients who received neoadjuvant chemotherapy (NAC), the influence of smoking status on the pathological response and prognosis remains unclear. This pooled analysis aimed to investigate whether smoking status is an independent risk factor for pathological response, recurrence, and prognosis in patients with bladder cancer who undergo NAC.

Methods: We searched PubMed, Web of Science, Embase, Cochrane Library, and Google Scholar for related studies published between 1990 and 2017. In total, 10 studies comprising 1,382 patients with muscleinvasive bladder cancer were included. The odds ratio (OR) and $95 \%$ confidence interval (CI) of complete pathological response, partial pathological response, overall survive (OS), recurrence, and cancer-specific mortality (CSM) were chosen as outcome measures. Analyses were performed using Review Manager (version 5.3, The Cochrane Collaboration, UK) and Stata statistical software (version 15, Stata Corp., USA).

Results: Compared to nonsmokers, smokers were less likely to have a complete pathologic response (OR $=0.55,95 \%$ CI: 0.35-0.87) and partial pathological response (OR =0.57, 95\% CI: 0.37-0.88). However, we found no significant association between smoking status and overall survival (OR =0.71, 95\% CI: 0.28-1.80), recurrence (OR $=1.35,95 \% \mathrm{CI}: 0.97-1.88)$, and cancer-specific mortality (OR =0.90, 95\% CI: 0.62-1.32).

Conclusions: Smoking reduces both complete and partial pathological response rate to NAC in patients with bladder cancer. Thus, smoking status should be given more importance when developing treatment plans and evaluating efficacy, particularly of NAC, among bladder cancer patients.
\end{abstract}

Keywords: Bladder cancer; neoadjuvant chemotherapy (NAC); smoking status; pathological response; pooled analysis

Submitted Jul 11, 2020. Accepted for publication Nov 04, 2020.

doi: $10.21037 /$ tau-20-1086

View this article at: http://dx.doi.org/10.21037/tau-20-1086

\section{Introduction}

Bladder cancer is now widely recognized as a threat to global development. In 2017, 474,000 and 197,000 individuals newly developed bladder cancer and died from the malignancy, respectively (1). Bladder cancer causes more than 3 billion disability-adjusted life years (1). Neoadjuvant chemotherapy (NAC) before radical cystectomy (RC) has been established as a better treatment modality based on level 1 evidence (2). Patients after NAC have improved 
better survival rates than patients who did not undergo NAC (3). Pathological downstaging for patients who undergo NAC can be a surrogate factor for the outcome of the patients and chemosensitivity (4). Despite the adverse health effect of cigarette smoking, particularly its carcinogenic effects, the World Health Organization reported that there are still 1.1 billion individuals who smoke. Smoking is the most common risk factor for bladder cancer (BC), accounting for more than $50 \%$ of $\mathrm{BC}$ cases $(5,6)$. Tobacco smoke carcinogens play a critical role in bladder cancer development (5); accordingly, smoking cessation can improve the outcomes of patients with bladder cancer $(7,8)$. However, there have been few studies about the influence of cigarette smoking on the efficacy of NAC, and the results are conflicting. Some studies showed that cigarette smoking was significantly associated with adverse pathological response in patients treated with NAC followed by RC (9). Further, compared with former smoking and never smoking status, current smoking was the strongest risk factor for disease recurrence (9). In contrast, some researchers argued that smoking status and complete pathologic response were not significantly associated from a statistical point of view (10). Thus, given these conflicting findings, it remains unclear whether smoking status influences the pathological response and oncological outcomes in bladder cancer patients treated with NAC followed by RC. This pooled analysis aimed to investigate whether smoking status is an independent risk factor for pathological response, recurrence, and prognosis in bladder cancer patients who undergo NAC. We present the following article in accordance with the PRISMA reporting checklist (available at http://dx.doi.org/10.21037/tau-201086).

\section{Methods}

\section{Study design and search strategy}

This study was conducted in accordance with the PRISMA guideline, and the study protocol of this pooled review was registered in the PROSPERO (International Prospective Register of Systematic Reviews). We searched PubMed, Web of Science, Embase, Cochrane Library, and Google Scholar for related articles published until November 2019 using the MeSH) keywords "Neoplasm, Urinary Bladder," "Neoadjuvant Therapies", and "Smoking Behaviors." The study selection flowchart is shown in Figure 1. The references of the identified studies were also reviewed manually to further identify any related studies.

\section{Selection criteria}

We selected articles based on the following four criteria (PICOS principle). First, the study population should include bladder cancer patients treated with neoadjuvant therapy followed by RC. Second, for intervention and comparison, information about smoking status should be included. Third, for outcomes, the pathological response or oncological outcomes including overall survival (OS), recurrence, or cancer-specific mortality (CSM) should be reported. Fourth, the study should be a cohort or casecontrol study. In total, 2001 articles were initially reviewed after removing duplicates. Eventually, 10 articles that met the inclusion criteria were included in the study; of these, 9 articles were retrospective studies and 1 was a phase II trial.

\section{Data extraction and quality assessment}

Two authors (HD and DQ) independently collected the data. These included title, first author, year of publication, number of patients, smoking status, chemotherapy regimens, number of patients with different smoking status and pathological outcomes, odds ratio (OR) of OS, recurrence, and cancer-specific mortality. Two authors (JH and DQ) separately assessed the quality of the studies following the Newcastle-Ottawa scale (NOS) (11) (Table S1). The study quality was assessed according to three parameters: cohort selection, cohort comparability, and outcome assessment. Higher scores reflect higher quality.

\section{Statistical analysis}

ORs were pooled to create a forest plot comparing pathological response, OS, recurrence, and CSM for different smoking status in muscle-invasive bladder cancer (MIBC) patients. The $\mathrm{I}^{2}$ test was used to estimate study variance. If the $\mathrm{I}^{2}<50 \%$, a fixed effect model was used; otherwise, a random effect model was used. Publication bias was evaluated using Egger's test, Begg's test, and funnel plots. Subgroup analyses according to smoking status (current smoking, former smoking, and never smoking) were also performed. All analyses were conducted using Review Manager version 5.3 (The Nordic Cochrane Centre, The Cochrane Collaboration, Copenhagen) and Stata statistical software (version 15). 

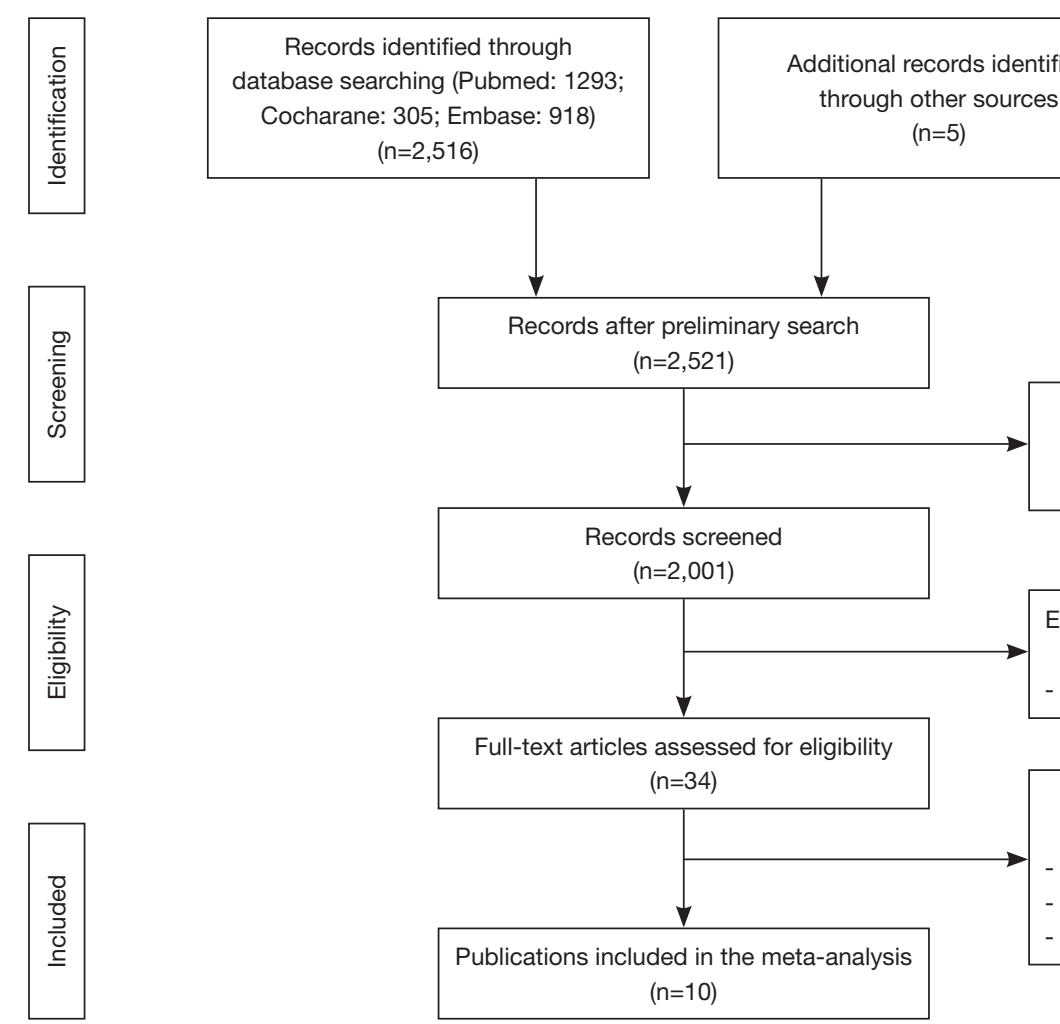

Exclusion after duplicates removed $(n=520)$

Exclusion after title and abstract screen $(n=1,967)$

All not relevant to our topic

Studies excluded after full text evaluation $(n=24)$ - Not including smoking status $(n=6)$ - Other study type $(n=8)$ - Insufficient data $(n=10)$

Figure 1 Study selection flowchart.

\section{Results}

\section{Search results and patient characteristics}

In total, 1,382 patients from 10 studies were included in this pooled analysis $(9,10,12-19)$. With respect to the followup time, most of the patients reached their end point. The majority of the patients included in our analysis were male $(89 \%)$ from North America. All the patients were pathologically diagnosed with MIBC from T2N0M0 to T4N0M0. We make separate analysis because not all studies contain the data of both complete response and partial response. The characteristics of the included articles are shown in Table 1. All the results are shown in Table 2.

\section{Smoking status as predictor of pathological response to NAC}

The pathological response including complete response (pT0) and partial response (anything less than pT2) for smokers and nonsmokers are shown as forest plots in Figure 2. Because there was low heterogeneity $\left(\mathrm{I}^{2}<50 \%\right)$ in complete and partial pathological response analyses, fixed-effect models were used in both analyses. The results showed a strong connection between smoking status and pathological response, both in complete and partial response. Smokers had a two-fold higher risk of not achieving pathological response. Four of six studies showed that any smoking status (current or former) negatively influenced patient outcomes. The pooled OR was 0.55 (95\% CI: $0.35-0.87, \mathrm{P}=0.008)$ for smokers versus nonsmokers (Figure 2A). With respect to partial response, three of four studies indicated an undesirable outcome for patients who were smokers. The pooled OR from the four studies was 0.57 (95\% CI: $0.37-0.88$ ) for the comparison between smokers and nonsmokers (Figure 2B). Three studies stratified the smoking status into three types as current, former, and nonsmokers. Subgroup analysis of former smokers compared to nonsmokers showed that former smoking status had negative effects on complete response $(\mathrm{OR}=0.53$, 95\% CI: 0.28-0.98). Meanwhile, subgroup analysis of current smokers versus nonsmokers showed that although current smoking status did not have a statistically significant impact on disease prognosis $(\mathrm{OR}=0.53,95 \% \mathrm{CI}$ : 0.28-1.02), smoking tended to have a negative influence on outcomes (Figure 2C). The same tendency was found 


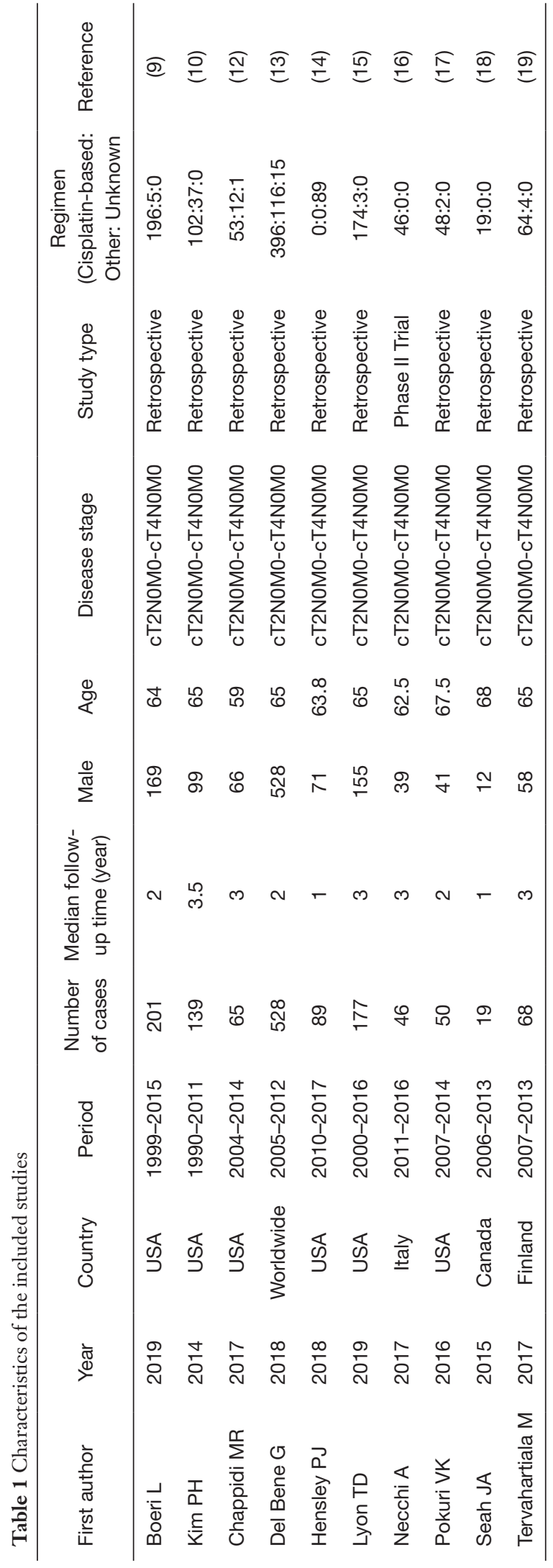

for partial response. Compared with nonsmokers, current smokers were less likely to achieve partial response (OR $=0.38,95 \%$ CI: $0.21-0.69)$, and the outcome was similar when former smokers were compared to nonsmokers, although the difference was not significant ( $\mathrm{OR}=0.59,95 \%$ CI: 0.35-1.01) (Figure 2D).

\section{Overall survival and smoking status}

The heterogeneity $\left(\mathrm{I}^{2}<50 \%\right)$ was low in OS analyses. Hence, a fixed-effect model was used. The results showed that smoking status was not correlated to the OS of bladder cancer patients who undergo NAC (OR $=0.71,95 \% \mathrm{CI}$ : $0.28-1.80$, Figure $3 A$ ). In addition, another two studies that compared between current smokers and nonsmokers showed that there is no evidence of association between smoking status and OS (OR $=1.10,95 \%$ CI: $0.77-1.55)$. Similarly, there was also no statistical evidence of former smoking having an influence on OS compared to nonsmoking (OR $=0.87$, 95\% CI: 0.64-1.18) (Figure 3B).

\section{Influence of smoking status on recurrence and CSM}

Two studies investigated the association between smoking status and recurrence or CSM. No obvious heterogeneity $\left(\mathrm{I}^{2}<50 \%\right)$ was observed in recurrence and CSM analyses. Thus, we selected fixed-effect models to analyze the data. With regard to recurrence, two studies reported a pooled OR of 1.41 (95\% CI: $0.86-2.33$ ) for current smokers and a pooled OR of 1.30 (95\% CI: 0.83-2.02) for former smokers (Figure 4A). The impact of smoking was mitigated. For CSM, it was not influenced by smoking status among current smokers compared to nonsmokers $(\mathrm{OR}=1.12$, 95\% CI: 0.64-1.96). Similar results were obtained in the comparison between former smokers and nonsmokers (OR $=0.75,95 \%$ CI: 0.45-1.26) (Figure 4B).

\section{Publication bias}

Publication bias was calculated using Begg's test and Egger's test for complete pathological response and partial pathological response for smokers and nonsmokers in more than four studies. We found a possible bias for complete pathological response [Begg, $\mathrm{P}=0.024$ (Figure S1A); Egger, $\mathrm{P}=0.007$ (Figure S1B)]. Meanwhile, for partial pathological response, both tests $[\mathrm{Begg}, \mathrm{P}=0.806$ (Figure S1C); Egger, $\mathrm{P}=0.298$ (Figure S1D)] showed that there was no publication bias. For further analysis about 
Table 2 Analysis of the influence of smoking on the oncologic outcomes of bladder cancer

\begin{tabular}{|c|c|c|c|c|c|c|}
\hline Outcomes & $\begin{array}{l}\text { No. of } \\
\text { studies }\end{array}$ & \multicolumn{2}{|c|}{ No. of patients (events*) } & Pooled OR (95\% Cl) & $\mathrm{P}$ & $I^{2}(\%)$ \\
\hline рT0 & 6 & 538 (119) & $137(42)$ & $0.53(0.33-0.85)$ & 0.008 & 0 \\
\hline рT2 & 4 & 486 (189) & $122(60)$ & $0.57(0.37-0.88)$ & 0.01 & 10 \\
\hline os & 2 & 85 (NA) & 29 (NA) & $0.71(0.28-1.80)$ & 0.47 & 4 \\
\hline CSM & 2 & 247 (NA) & 93 (NA) & $0.90(0.62-1.32)$ & 0.59 & 0 \\
\hline
\end{tabular}

NA: we cannot extract directly or estimate indirectly the number of events from the original article; events*: the pathological response or oncologic outcomes such as overall survival, recurrence and cancer specific mortality. pT0, pathological complete response; pT2, pathological partial response; OS, overall survival; Re, recurrence; CSM, cancer-specific mortality.

A

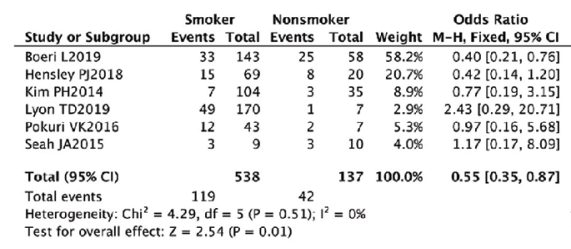

Heterogeneity: Chi' $=4.29$, df $=5(P=0.52) ; 1^{2}=0.4$
Test for overall effect: $Z=2.54(P=0.01)$.

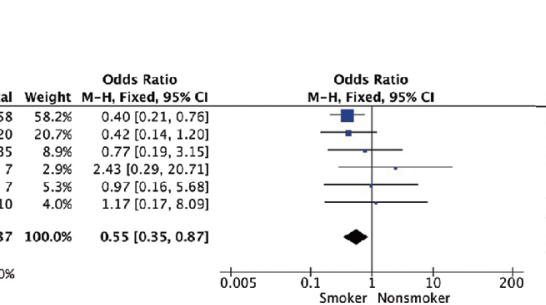

B

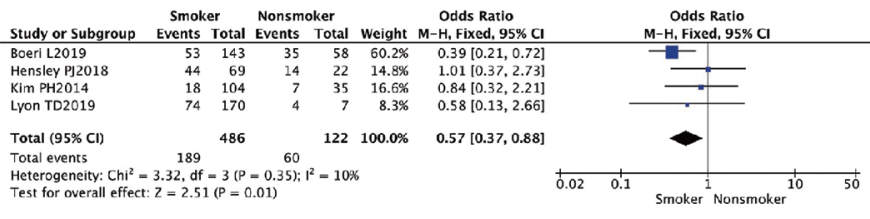

C
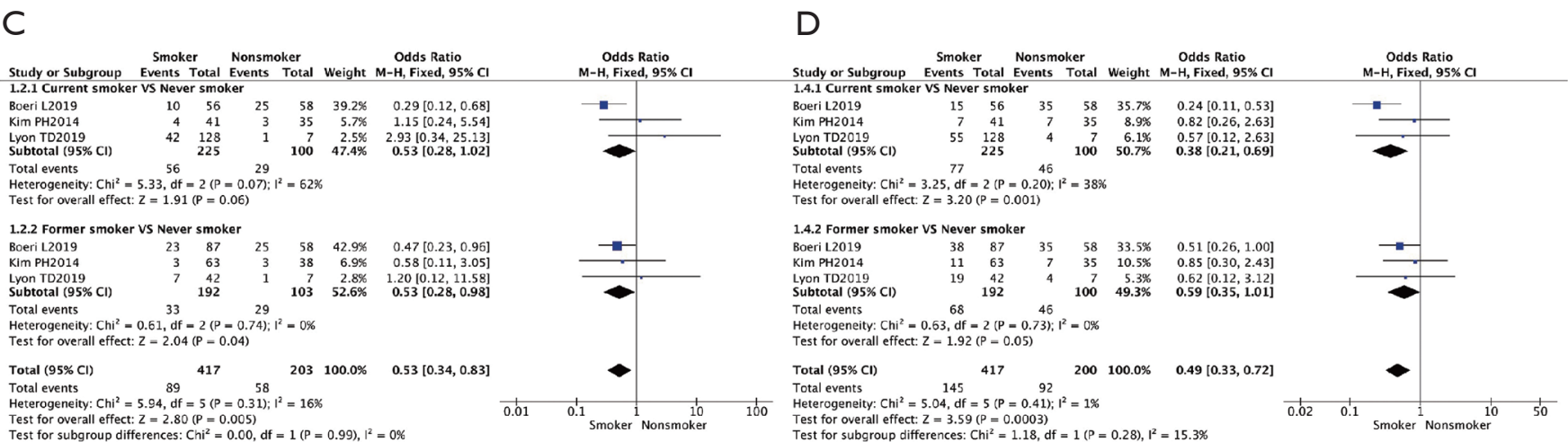

Figure 2 Pooled analysis of studies that examined the associations between smoking and pathological response. (A) Complete response (smoker vs. nonsmoker). (B) Partial response (smoker vs. nonsmoker). (C) Complete response (current smoker vs. nonsmoker and former smoker vs. nonsmoker). (D) Partial response (current smoker vs. nonsmoker and former smoker vs. nonsmoker).

the possible bias for complete pathological response, we conducted a sensitivity analysis (Figure S2).

\section{Discussion}

Previous studies have reported that smoking behavior potentially affects the pathological outcome of patients with bladder cancer $(5,6)$ and cause complications among various cancers $(20,21)$. Tobacco products are among the at least 70 known carcinogens worldwide (22). Nicotine, the major component of cigarettes, affects the occurrence and outcomes of numerous cancer types through different pathways $(23,24)$. Nicotine strongly activates Stat3, leading to cyclin D1 overexpression, cell cycle perturbations, and chemoresistance. Moreover, nicotine initiates Stat3 signaling, resulting in the loss of extracellular signalregulated protein kinase 1/2 (ERK 1/2) activation and reduced chemosensitivity via nicotinic acetylcholine 
A

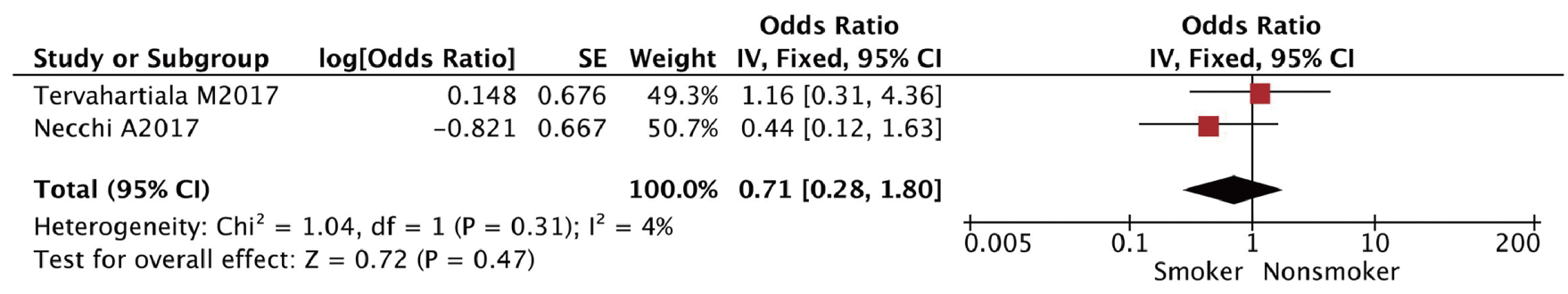

B

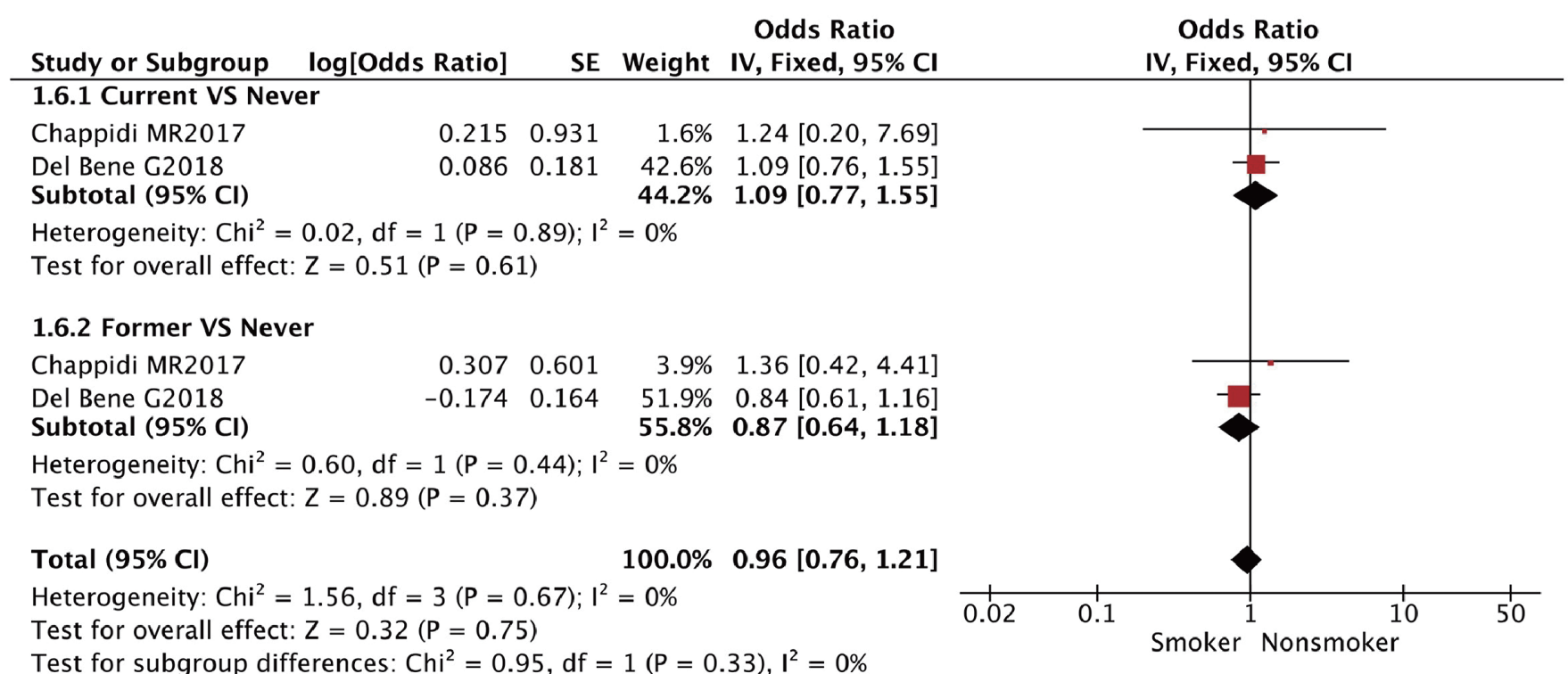

Figure 3 Pooled analysis of studies that examined the associations between smoking and overall survival (OS). (A) Smoker vs. nonsmoker (B) Current smoker vs. nonsmoker and former smoker vs. nonsmoker.
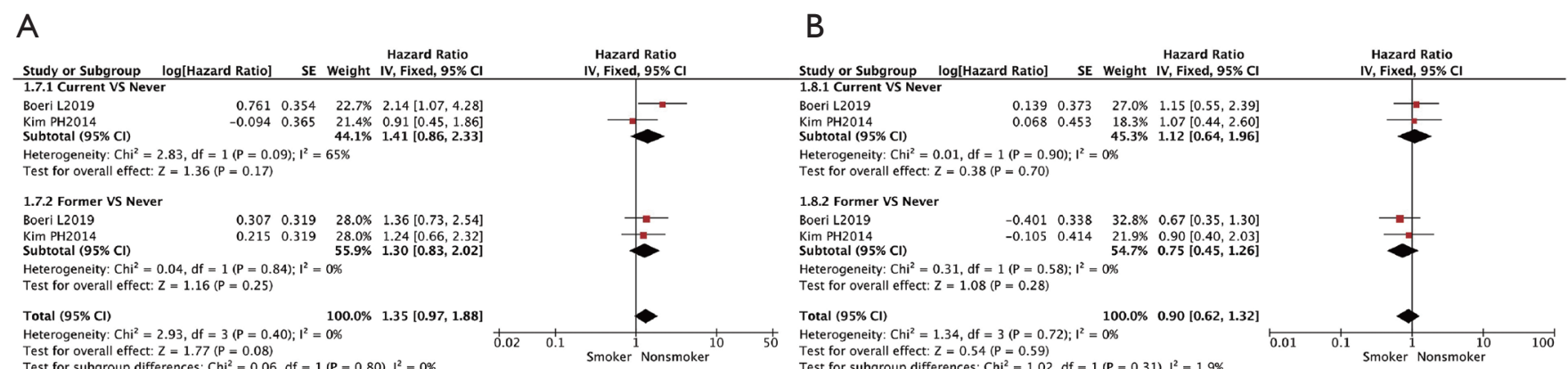

Figure 4 Pooled analysis of studies that examined the associations of smoking with (A) recurrence and (B) cancer-specific mortality (CSM). 
receptors and b-adrenoceptors (23). In addition, known carcinogenic compounds such as aromatic amines and $\mathrm{N}$-nitroso compounds can directly damaging doublestranded, base modifications of DNA, thus potentially influencing survival after neoadjuvant therapy (25).

There is sufficient evidence supporting that cigarette smoking is associated with the development of various cancers (26-29). All forms of tobacco smoking increase the risk of cancer development, and the components of cigarette influence the efficacy of chemotherapy drugs $(23,30)$. Smoking can also negatively affect oncological outcomes among patients who undergo neoadjuvant therapy (21). However, although the association between smoking status and lung cancer has already been established (31), there has been no systematic analysis on the impact of smoking on the outcomes of bladder cancer patients who underwent neoadjuvant therapy, partly because of insufficient data. Two studies on the association between smoking status and NAC in bladder cancer obtained contrasting findings $(9,10)$. One revealed that smoking behavior was significantly associated with adverse pathological response, and smokers had a higher risk of no response to NAC than never smokers (9). In contrast, the other one did not find any significant associations between smoking and pathologic response or cancer-specific outcomes after NAC and RC (10).

In the current pooled analysis, we analyzed the OR of complete pathological response (pT0) and partial pathological response (anything less than pT2) for two smoking status (smokers and nonsmokers) and three smoking status (current smokers, former smokers, and nonsmokers). The results supported that smoking status influences complete pathological response and partial pathological response in these patients. Compared to nonsmokers, smokers had nearly two times higher risk of poor outcomes. To the best of our knowledge, this is the first pooled analysis to focus on the association between smoking status and prognosis of bladder cancer patients treated with NAC.

The patients who achieve complete response will obviously achieve a better outcome than those without complete response. Even partial response could still be beneficial (4). Because treatment response to neoadjuvant therapy is a crucial influencing factor of survival outcomes, the association between smoking status and the prognosis of patients who undergo neoadjuvant therapy indicates that smoking behaviors probably lead to a worse pathological response (9).

Our study investigated the association between OS and smoking status. Compared to studies on pathological response, there are considerably fewer studies focusing on the role of smoking in recurrence because its influence is yet to be acknowledged. Further, results have been conflicting. Whereas some studies on bladder cancer reported that current smoking is an independent factor for recurrence (9), some studies indicated no association between smoking status and recurrence (10). Two studies included in this pooled analysis reported no significant association between smoking and recurrence, regardless of current or former smoker status. More studies with a large sample size and longer follow-up time are needed to clearly establish the influence of smoking status on the risk of recurrence. Further research will be helpful to better understand the impact of smoking on bladder cancer pathogenesis.

Most cancers can be influenced by smoking status. One study reported that the incidence, mortality, and prognosis of bladder cancer are associated with smoking status (32). However, there is no evidence indicating an association between smoking status and bladder cancer treated with neoadjuvant therapy to date. This is because most studies lack a more detailed analysis of smoking behaviors (e.g., intensity and duration), as some researchers have already reported that the impact of cigarette smoking is cumulative, and cigarette exposure lasting more than 20 years can significantly change the outcome. In the current pooled analysis, despite the small number of studies, smoking behaviors were found to be significantly associated with a higher rate of mortality. This could be because of several reasons. First, most of our included studies are from North America, which could have led to a geographical bias, because of the race $(4,33)$. Second, cancer histology can also influence survival outcomes in patients undergoing neoadjuvant therapy. Those with pure urothelial carcinoma have better survival than patients with mixed tumor type. One study included in the current pooled analysis showed that patients with pure urothelial bladder cancer have 10 times better survival outcomes than those with mixed type bladder cancer $(17,34)$. Body mass index also influenced the outcome of our analysis. Obese patients showed a higher risk of developing bladder cancer (35).

There are several limitations in our study. First, the number of patients in some of the analyses was limited. Second, most of the included studies evaluated obtain smoking status using interviews, questionnaires, and patient records; thus, the possibility of information bias could not be eliminated. Despite these limitations, we believe that our pooled analysis is valuable because to our best knowledge, 
this is the first study to report that smoking status can influence the pathological response to NAC in bladder patients and lead to a worse prognosis. Further welldesigned studies with large population size are warranted to verify the association between smoking status and the prognosis of bladder cancer patients who undergo NAC.

\section{Conclusions}

Smoking has a negative impact on the prognosis of bladder cancer patients who underwent NAC, limiting its efficacy with respect to pathological responses. Further, although smoking was not significantly correlated with OS, recurrence, and CSM, there was a tendency toward an increased risk for worse outcomes among smokers. Thus, smoking status should be given more importance when developing treatment plans and evaluating efficacy, particularly of NAC, among bladder cancer patients.

\section{Acknowledgments}

Funding: This work was supported by the National Natural Science Foundation of China (81572523, 81700665, 81873626, 81902592), the Hunan Province Funds for Distinguished Young Scientists of China (2016JJ1026), Hunan Province Key R\&D Program (2019SK2202), Hunan Province Technology Innovation Guidance Program Clinical Medical Technology Innovation Guidance Project No.2018SK51714, and Xiangya Hospital Youth Fund (2018Q09).

\section{Footnote}

Reporting Checklist: The authors have completed the PRISMA reporting checklist. Available at http://dx.doi. org/10.21037/tau-20-1086

Peer Review File: Available at http://dx.doi.org/10.21037/ tau-20-1086

Conflicts of Interest: All authors have completed the ICMJE uniform disclosure form (available at http://dx.doi. org/10.21037/tau-20-1086). Dr. XZ serves as an unpaid editorial board member of Translational Andrology and Urology from Mar 2015 to Feb 2021. The other authors have no conflicts of interest to declare.

Ethical Statement: The authors are accountable for all aspects of the work in ensuring that questions related to the accuracy or integrity of any part of the work are appropriately investigated and resolved.

Open Access Statement: This is an Open Access article distributed in accordance with the Creative Commons Attribution-NonCommercial-NoDerivs 4.0 International License (CC BY-NC-ND 4.0), which permits the noncommercial replication and distribution of the article with the strict proviso that no changes or edits are made and the original work is properly cited (including links to both the formal publication through the relevant DOI and the license). See: https://creativecommons.org/licenses/by-nc-nd/4.0/.

\section{References}

1. Global Burden of Disease Cancer Collaboration, Fitzmaurice C, Abate D, et al. Global, Regional, and National Cancer Incidence, Mortality, Years of Life Lost, Years Lived With Disability, and Disability-Adjusted LifeYears for 29 Cancer Groups, 1990 to 2017: A Systematic Analysis for the Global Burden of Disease Study. JAMA Oncol 2019;5:1749-68.

2. Alfred Witjes J, Lebret T, Comperat EM, et al. Updated 2016 EAU Guidelines on Muscle-invasive and Metastatic Bladder Cancer. Eur Urol 2017;71:462-75.

3. Fahmy O, Khairul-Asri MG, Schubert T, et al. A systematic review and meta-analysis on the oncological long-term outcomes after trimodality therapy and radical cystectomy with or without neoadjuvant chemotherapy for muscle-invasive bladder cancer. Urol Oncol 2018;36:43-53.

4. Rosenblatt R, Sherif A, Rintala E, et al. Pathologic Downstaging Is a Surrogate Marker for Efficacy and Increased Survival Following Neoadjuvant Chemotherapy and Radical Cystectomy for Muscle-Invasive Urothelial Bladder Cancer. Eur Urol 2012;61:1229-38.

5. Jin F, Thaiparambil J, Donepudi SR, et al. TobaccoSpecific Carcinogens Induce Hypermethylation, DNA Adducts, and DNA Damage in Bladder Cancer. Cancer Prev Res (Phila) 2017;10:588-97.

6. Burger M, Catto JW, Dalbagni G, et al. Epidemiology and risk factors of urothelial bladder cancer. Eur Urol 2013;63:234-41.

7. Toll BA, Brandon TH, Gritz ER, et al. Assessing tobacco use by cancer patients and facilitating cessation: an American Association for Cancer Research policy statement. Clin Cancer Res 2013;19:1941-8.

8. Fernandez MI, Brausi M, Clark PE, et al. Epidemiology, 
prevention, screening, diagnosis, and evaluation: update of the ICUD-SIU joint consultation on bladder cancer. World J Urol 2019;37:3-13.

9. Boeri L, Soligo M, Frank I, et al. Cigarette smoking is associated with adverse pathological response and increased disease recurrence amongst patients with muscle-invasive bladder cancer treated with cisplatinbased neoadjuvant chemotherapy and radical cystectomy: a single-centre experience. BJU Int 2019;123:1011-9.

10. Kim PH, Kent M, Zhao P, et al. The impact of smoking on pathologic response to neoadjuvant cisplatin-based chemotherapy in patients with muscle-invasive bladder cancer. World J Urol 2014;32:453-9.

11. Stang A. Critical evaluation of the Newcastle-Ottawa scale for the assessment of the quality of nonrandomized studies in meta-analyses. Eur J Epidemiol 2010;25:603-5.

12. Chappidi MR, Kates M, Brant A, et al. Assessing Cancer Progression and Stable Disease After Neoadjuvant Chemotherapy for Organ-confined Muscle-invasive Bladder Cancer. Urology 2017;102:148-58.

13. Del Bene G, Calabro F, Giannarelli D, et al. Neoadjuvant vs. Adjuvant Chemotherapy in Muscle Invasive Bladder Cancer (MIBC): Analysis From the RISC Database. Front Oncol 2018;8:463.

14. Hensley PJ, Goodwin J, Davenport DL, et al. Optimization of Patient Selection for Neoadjuvant Chemotherapy in Muscle-invasive Urothelial Carcinoma of the Bladder. Clin Genitourin Cancer 2018;16:e851-8.

15. Lyon TD, Frank I, Takahashi N, et al. Sarcopenia and Response to Neoadjuvant Chemotherapy for MuscleInvasive Bladder Cancer. Clin Genitourin Cancer 2019;17:216-22.e5.

16. Necchi A, Lo Vullo S, Raggi D, et al. Neoadjuvant sorafenib, gemcitabine, and cisplatin administration preceding cystectomy in patients with muscle-invasive urothelial bladder carcinoma: An open-label, single-arm, single-center, phase 2 study. Urol Oncol 2018;36:8 e1-8 e.

17. Pokuri VK, Syed JR, Yang Z, et al. Predictors of Complete Pathologic Response (pT0) to Neoadjuvant Chemotherapy in Muscle-invasive Bladder Carcinoma. Clin Genitourin Cancer 2016;14:e59-65.

18. Seah JA, Leibowitz-Amit R, Atenafu EG, et al. Neutrophil-Lymphocyte Ratio and Pathological Response to Neoadjuvant Chemotherapy in Patients With MuscleInvasive Bladder Cancer. Clin Genitourin Cancer 2015;13:e229-33.

19. Tervahartiala M, Taimen P, Mirtti T, et al. Immunological tumor status may predict response to neoadjuvant chemotherapy and outcome after radical cystectomy in bladder cancer. Sci Rep 2017;7:12682.

20. Quan H, Ouyang L, Zhou H, et al. The effect of preoperative smoking cessation and smoking dose on postoperative complications following radical gastrectomy for gastric cancer: a retrospective study of 2469 patients. World J Surg Oncol 2019;17:61.

21. Schaverien MV, Munnoch DA. Effect of neoadjuvant chemotherapy on outcomes of immediate free autologous breast reconstruction. Eur J Surg Oncol 2013;39:430-6.

22. Khariwala SS, Hatsukami D, Hecht SS. Tobacco carcinogen metabolites and DNA adducts as biomarkers in head and neck cancer: potential screening tools and prognostic indicators. Head Neck 2012;34:441-7.

23. Chen RJ, Ho YS, Guo HR, et al. Long-term Nicotine Exposure-Induced Chemoresistance Is Mediated by Activation of Stat3 and Downregulation of ERK1/2 via nAChR and Beta-Adrenoceptors in Human Bladder Cancer Cells. Toxicol Sci 2010;115:118-30.

24. Dasgupta P, Kinkade R, Joshi B, et al. Nicotine inhibits apoptosis induced by chemotherapeutic drugs by upregulating XIAP and survivin. Proc Natl Acad Sci U S A 2006;103:6332-7.

25. Cumberbatch MGK, Jubber I, Black PC, et al. Epidemiology of Bladder Cancer: A Systematic Review and Contemporary Update of Risk Factors in 2018. Eur Urol 2018;74:784-95.

26. Pickles T, Liu M, Berthelet E, et al. The effect of smoking on outcome following external radiation for localized prostate cancer. J Urol 2004;171:1543-6.

27. Rieken M, Shariat SF, Kluth LA, et al. Association of Cigarette Smoking and Smoking Cessation with Biochemical Recurrence of Prostate Cancer in Patients Treated with Radical Prostatectomy. Eur Urol 2015;68:949-56.

28. Wen S, Ren W, Xue B, et al. Prognostic factors in patients with penile cancer after surgical management. World $\mathrm{J}$ Urol 2018;36:435-40.

29. Zeng J, Tang Y, Wu P, et al. Alcohol consumption, tobacco smoking, betel quid chewing and oral health associations with hypopharyngeal cancer among men in Central South China: a case-control study. Cancer Manag Res 2019;11:6353-64.

30. Samanta D, Kaufman J, Carbone DP, et al. Long-term smoking mediated down-regulation of Smad3 induces resistance to carboplatin in non-small cell lung cancer. Neoplasia 2012;14:644-55.

31. Hasegawa Y, Ando M, Maemondo M, et al. The role 
of smoking status on the progression-free survival of non-small cell lung cancer patients harboring activating epidermal growth factor receptor (EGFR) mutations receiving first-line EGFR tyrosine kinase inhibitor versus platinum doublet chemotherapy: a meta-analysis of prospective randomized trials. Oncologist 2015;20:307-15.

32. Wyszynski A, Tanyos SA, Rees JR, et al. Body mass and smoking are modifiable risk factors for recurrent bladder cancer. Cancer 2014;120:408-14.

33. Janisch F, Shariat SF, Schernhammer E, et al. The interaction of gender and smoking on bladder cancer risks.

Cite this article as: $\mathrm{He}$ T, Hu J, Qiu D, Deng H, Hu J, Chen J, $\mathrm{Zu} \mathrm{X}$. Smoking status and pathological response to neoadjuvant chemotherapy among patients with bladder cancer: a pooled analysis. Transl Androl Urol 2021;10(1):374-383. doi: 10.21037/ tau-20-1086
Curr Opin Urol 2019;29:249-55.

34. Crabb S, Danson SJ, Catto JWF, et al. SPIRE - combining SGI-110 with cisplatin and gemcitabine chemotherapy for solid malignancies including bladder cancer: study protocol for a phase Ib/randomised IIa open label clinical trial. Trials 2018;19:216.

35. Al-Zalabani AH, Stewart KFJ, Wesselius A, et al. Modifiable risk factors for the prevention of bladder cancer: a systematic review of meta-analyses. Eur J Epidemiol 2016;31:811-51. 


\section{Supplementary}

Table S1 Assessment of quality of studies by Newcastle-Ottawa Scale

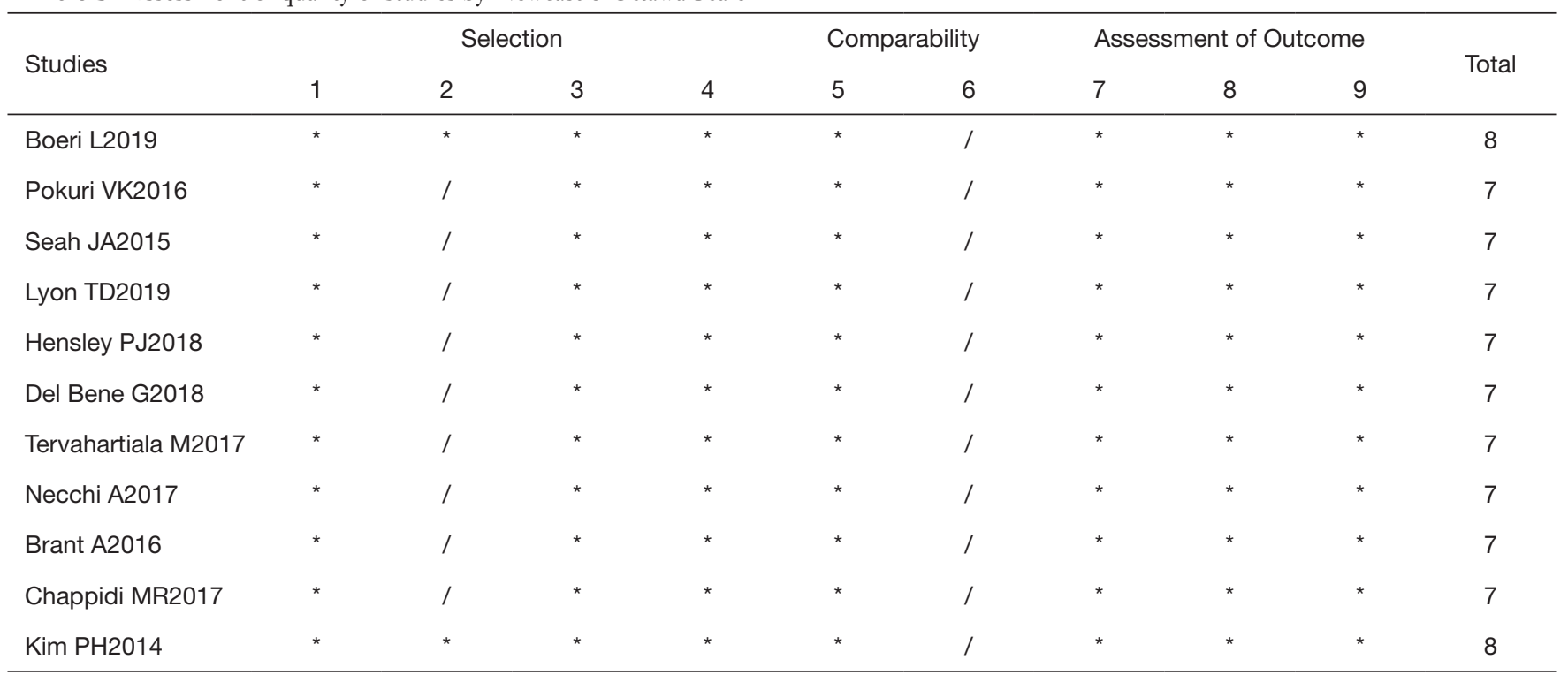

1 = Representativeness of the exposed cohort; 2 = Ascertainment of exposure; 3 = Selection of Controls; 4 = Demonstration that outcome of interest was not present at start of study; 5 = study controls for the most important factors; 6 = study controls for any additional factor; 7 = Assessment of outcome; 8 = Was follow-up long enough for outcomes to occur; 9 = Adequacy of follow up of cohort.

A

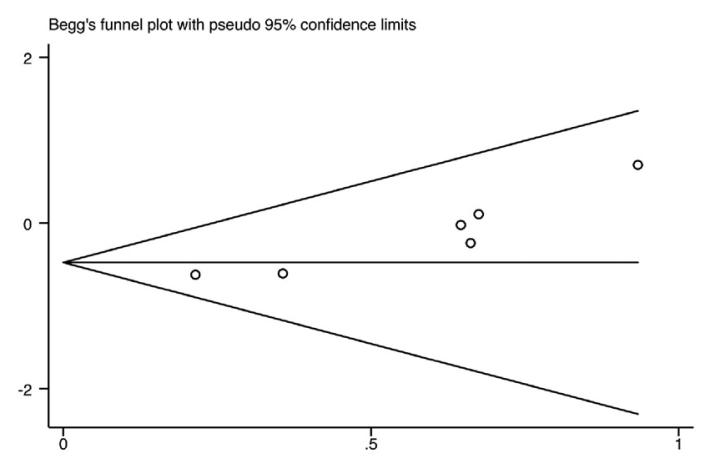

C

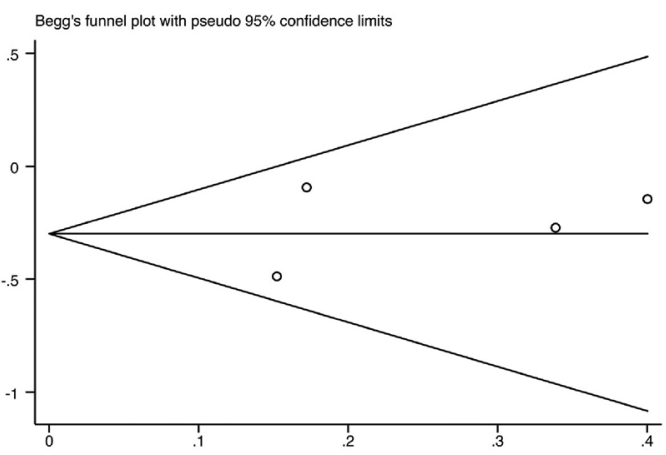

B

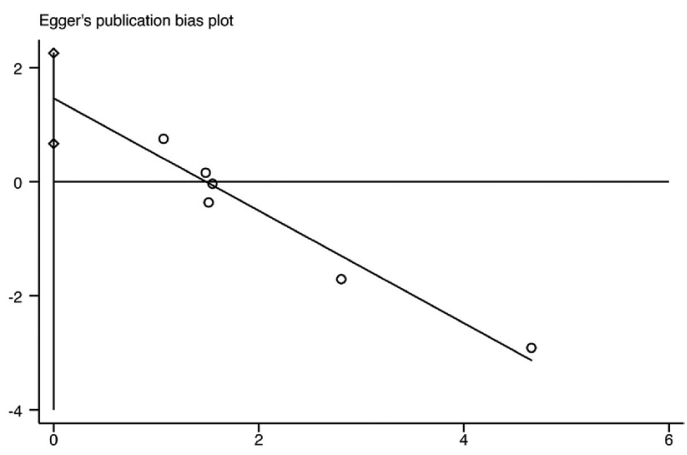

D

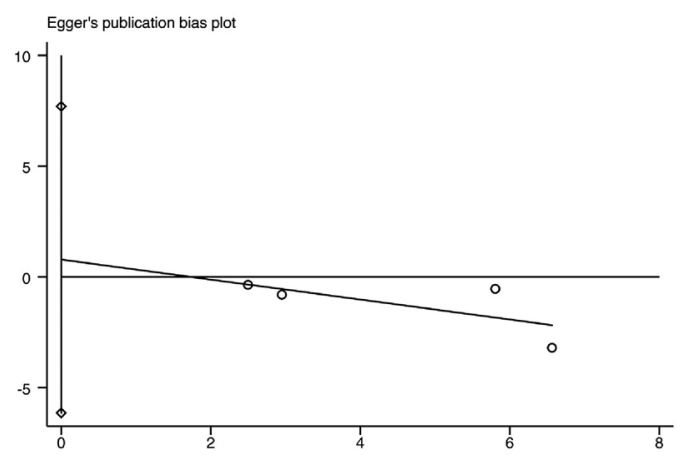

Figure S1 Pooled analysis of studies that examined the publication bias. (A) Begg's test for complete pathological response. (B) Egger's test for complete pathological response. (C) Begg's test for partial pathological response. (D) Begg's test for partial pathological response. 


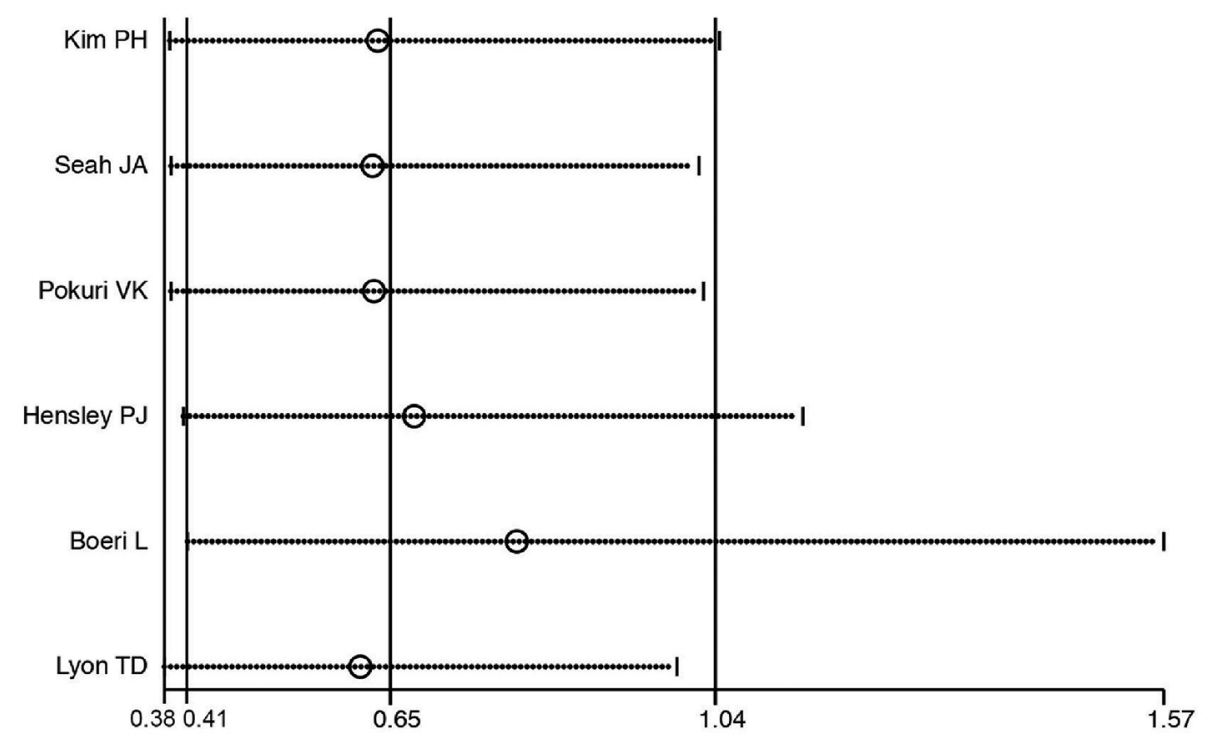

Figure S2 Sensitivity analysis of the publication bias for complete pathological response. CI: confidence interval; SE: standard error. 